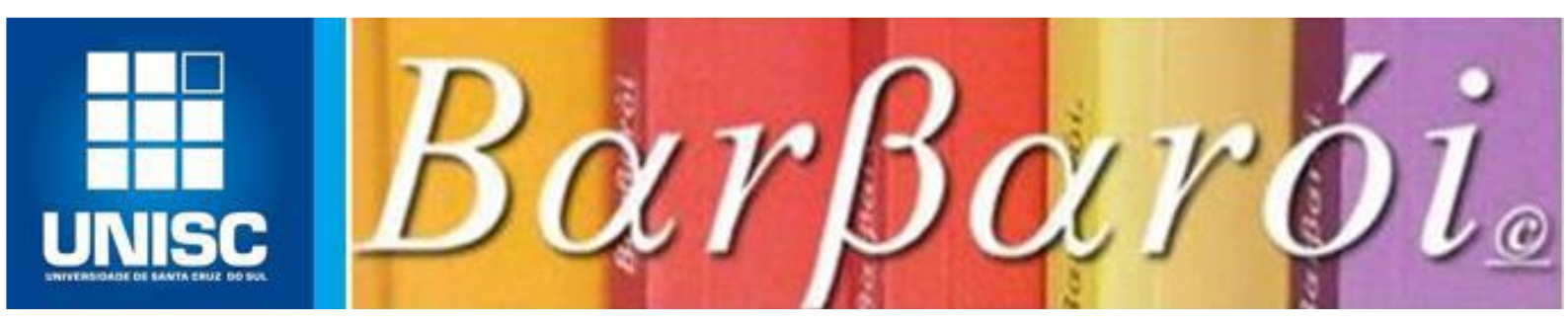

\title{
GRUPO DE TREINAMENTO DE HABILIDADES SOCIAIS EM UM CENTRO DE ATENÇÃO PSICOSSOCIAL
}

\section{DOI: http://dx.doi.org/10.17058/barbaroi.v0i0.12258}

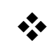 \\ Nilve Junges \\ Hospital de Clínicas de Porto Alegre - HCPA - Brasil \\ Flavia Pimentel Pereira \\ Hospital de Clínicas de Porto Alegre - HCPA - Brasil \\ Juliana Unis Castan \\ Hospital de Clínicas de Porto Alegre - HCPA - Brasil \\ $*$
}

\section{Resumo}

A assistência a usuários com transtorno mental no Brasil vem passando por diversas reformulações. Atualmente, esse público é atendido em Centro de Atenção Psicossocial (CAPS) por uma equipe multiprofissional que realiza o cuidado através de diferentes propostas terapêuticas. Este trabalho busca apresentar a experiência de um grupo de treinamento de habilidades sociais (THS) em um CAPS. O THS foi desenvolvido com 7 usuários com transtorno mental grave, teve duração de 12 sessões baseadas em cronograma de temáticas específicas e foi coordenado por dois profissionais, da enfermagem e psicologia. Este estudo é um relato de experiência baseado nos registros e observações dos profissionais. A participação dos usuários no grupo permitiu o desenvolvimento de autonomia, responsabilidade e assertividade diante de situações sociais. As características de grupo fechado e perfil semelhante permitiram a boa vinculação dos participantes e a formação de uma identidade grupal, características fundamentais para a proposta do THS. Como fatores limitadores, identificou-se a diferença na capacidade cognitiva de dois participantes que demandaram maior flexibilização e tempo para algumas propostas. A realização da proposta de THS com usuários com transtorno mental grave em um CAPS mostrou-se efetiva, atingindo os objetivos propostos e configurando-se como uma prática clínica inovadora no contexto da saúde mental coletiva.

Palavras-chave: Habilidades Sociais; Saúde Mental; Serviços de Saúde Mental; 


\section{Introdução}

Na história da atenção à Saúde Mental no Brasil, o período que antecedeu a Reforma Psiquiátrica caracterizou-se pelo modelo manicomial, o qual preconizava uma abordagem centrada na doença. Os sujeitos em sofrimento psíquico eram privados do convívio comunitário, institucionalizados e, diante disso, restringiam-se as possibilidades de tratamento e reintegração ao meio social. Esse modelo teve como marco a ineficácia dos tratamentos e, em muitos casos, até a violação dos direitos humanos, ao considerarmos seu caráter de aprisionamento e a violência institucional (AMARANTE, 2007).

Diante deste cenário, no final da década de 70, iniciaram no Brasil uma série de encontros e discussões sobre a loucura, o modelo manicomial de 'tratamento' e os efeitos da instituição asilar. Assim, surgiu no país o movimento antimanicomial, que visa a desinstitucionalização de pacientes psiquiátricos em situação asilar de longa data, a reinserção psicossocial destes e o cuidado humanizado à pessoa acometida por doença mental nos diferentes espaços de saúde. Dentre as propostas da Reforma Psiquiátrica foram criados diversos serviços, dentre eles o Centro de Atenção Psicossocial (CAPS). Trata-se de um espaço de tratamento, componente da Rede de Atenção Psicossocial (RAPS), para pessoas que sofrem com transtornos mentais graves e persistentes, cuja severidade justifique sua permanência em um dispositivo de cuidado intensivo, comunitário e personalizado. Tem como objetivo a reabilitação psicossocial, através da integração territorial, social e familiar, orientado por uma perspectiva de clínica ampliada de base comunitária e territorial e pautado nos princípios do Sistema Único de Saúde (SUS) (BRASIL, 2004).

As equipes dos CAPS são multiprofissionais e podem incluir enfermeiros, psicólogos, psiquiatras, terapeutas ocupacionais, profissionais de educação física, assistentes sociais, técnicos de enfermagem e outros técnicos. Atuando no âmbito da promoção da saúde e na prevenção de agravos, a equipe tem por tarefa formular propostas e projetos terapêuticos singulares que envolvam condutas e medidas terapêuticas visando reabilitação psicossocial através da construção da autonomia e melhora na qualidade de vida. Dentre as estratégias e modalidades de tratamento nos CAPS, os grupos terapêuticos recebem destaque, pois são um importante recurso no tratamento clínico e na reabilitação psicossocial.

Os sintomas dos transtornos psicóticos variam em expressão e intensidade. Muitas vezes, as alucinações e desorganização do pensamento cedem com o uso adequado dos psicofármacos. Entretanto, sintomas de embotamento e isolamento parecem ser mais persistentes, trazendo sofrimento e limitações no funcionamento diário dos usuários. 
Habilidades como estabelecer e manter amizades e relacionamentos amorosos, expressar necessidades, expor sentimentos e solicitar ajuda permeiam as dificuldades e queixas dos usuários. Essas habilidades, chamadas de habilidades sociais, tanto podem ter sido prejudicadas pelo desenvolvimento da doença, como podem não terem sido aprendidas, visto que transtornos mentais tipicamente se manifestam na adolescência ou início da idade adulta (SADOCK, SADOCK e RUIZ, 2016).

O grupo de Treinamento de Habilidades Sociais (THS) buscou desenvolver novas habilidades sociais, melhorar ou ampliar a frequência de habilidades existentes e diminuir comportamentos que vão de encontro a habilidades desejadas através de uma seleção de atividades planejadas e sistemáticas (DEL PRETTE e DEL PRETTE, 2011). Com base na teoria da aprendizagem social e no condicionamento operante, o THS visa avaliar e desenvolver habilidades emocionais, sociais, profissionais e familiares, com foco nas habilidades de comunicação e resolução de problemas. Assim, foi proposto um grupo de Treinamento de Habilidades Sociais para usuários que frequentam o CAPS e necessitam desenvolver e fortalecer habilidades sociais.

Este trabalho visa detalhar a construção e desenvolvimento de um grupo de Treinamento de Habilidades Sociais em um CAPS do sul do Brasil.

\section{Metodologia}

Este artigo busca descrever a experiência do desenvolvimento e condução do Grupo de Treinamento de Habilidades Sociais (THS), realizado em um CAPS da região sul do Brasil. O grupo foi conduzido pelos residentes multiprofissionais de saúde mental, dos núcleos de enfermagem e psicologia, e supervisionado pelas preceptoras destas áreas. Para o desenvolvimento desta atividade, além dos recursos pessoais, contou-se com sala espaçosa e arejada, computadores com acesso à internet, material gráfico e lúdico.

A indicação dos participantes iniciou com discussões na equipe sobre possíveis beneficiários, considerando estabilidade do quadro clínico, capacidade mínima de manter a atenção e seguir instruções, necessidade de desenvolver habilidades sociais, especialmente de comunicação, e desejo de participar. O grupo foi composto por 07 usuários que aceitaram participar da proposta, indicados em reunião de equipe de acordo com perfil, dificuldades e interesses. Os dados demográficos dos participantes foram organizados em forma de figura (Figura 1). 
Figura 1. Perfil dos participantes.

\begin{tabular}{|l|l|l|l|}
\hline Usuário & Sexo & Idade & Diagnósticos \\
\hline A & Masculino & 33 & Esquizofrenia e deficiência intelectual leve \\
\hline B & Masculino & 20 & Transtorno do espectro autista \\
\hline C & Masculino & 25 & $\begin{array}{l}\text { Esquizofrenia paranóide com predominância de } \\
\text { comportamentos compulsivos (rituais obsessivos) }\end{array}$ \\
\hline D & Masculino & 24 & Transtorno afetivo bipolar e deficiência intelectual leve \\
\hline E & Masculino & 28 & Transtorno esquizoafetivo e transtorno obsessivo \\
compulsivo
\end{tabular}

Assim, estabeleceu-se um grupo relativamente homogêneo com relação à idade, com exceção de uma usuária. A maioria dos usuários eram homens, o que está de acordo com a realidade atual do CAPS onde o grupo foi desenvolvido. Apesar dos diferentes diagnósticos, todos os usuários possuíam transtorno mental grave com dificuldade de inserção social, motivo pelo qual estavam em acompanhamento no CAPS.

\section{Procedimentos}

Foi estabelecido um grupo fechado, de 12 sessões, composto por 07 usuários. As sessões foram previamente estruturadas de forma flexível, possibilitando modificações e adaptações ao longo do processo. Os encontros tinham duração de uma hora, realizados uma vez por semana, no turno da manhã. O grupo contava com coordenador e co-coordenador. Baseado na literatura, nas observações dos terapeutas e nas verbalizações dos usuários, o plano de trabalho foi definido conforme Figura 2. 
Figura 2. Plano de Trabalho.

\begin{tabular}{|c|c|}
\hline Sessão & Conteúdo a trabalhar e objetivos \\
\hline 1.Introdução & $\begin{array}{l}\text { Apresentação dos integrantes; Proposta do treinamento e } \\
\text { introdução dos conceitos de habilidades sociais; Apresentação do } \\
\text { programa; Elaboração do Contrato }\end{array}$ \\
\hline $\begin{array}{l}\text { 2. Dificuldades e } \\
\text { Limitações; } \\
\text { Objetivos }\end{array}$ & $\begin{array}{l}\text { Análise de dificuldades e limitações, assim como facilidades, } \\
\text { identificando exemplos práticos e concretos da vida dos usuários; } \\
\text { Formulação de objetivos gerais do grupo e específico para cada } \\
\text { usuário }\end{array}$ \\
\hline $\begin{array}{l}\text { 3. Comunicação } \\
\text { Inicial }\end{array}$ & $\begin{array}{l}\text { Habilidades de fazer pedidos, aproximar-se de outros, pedir } \\
\text { informações na rua }\end{array}$ \\
\hline $\begin{array}{l}\text { 4. Habilidades } \\
\text { Corporais }\end{array}$ & Habilidades de olhar-se e olhar os outros: Postura, trejeitos, tiques \\
\hline 5. Conversação & Habilidades de conversação: iniciar, continuar e finalizar conversas \\
\hline 6. Leitura social & $\begin{array}{l}\text { Habilidades de perceber o ambiente: identificar pistas sociais } \\
\text { (sinais emitidos pelos outros/ambiente que sinalizam } \\
\text { prazer/desprazer, interesse/desinteresse e possibilitam modulação } \\
\text { na interação) }\end{array}$ \\
\hline $\begin{array}{l}\text { 7. Expressão } \\
\text { sentimentos }\end{array}$ & $\begin{array}{l}\text { Habilidades de expressão: Formas assertivas de expressar } \\
\text { sentimentos positivos e negativos, agrado e desagrado }\end{array}$ \\
\hline $\begin{array}{l}\text { 8. Pedidos e } \\
\text { expressão de } \\
\text { necessidades }\end{array}$ & $\begin{array}{l}\text { Habilidades de recorrer ao outro: Pedir ajuda, solicitar espaço, } \\
\text { emitir opinião, realizar trocas }\end{array}$ \\
\hline 9. Críticas & $\begin{array}{l}\text { Habilidades de lidar com críticas: examinar reações frente a } \\
\text { críticas, aprender a ouvir, formular e expressar críticas }\end{array}$ \\
\hline 10. Comunicação & Limpeza da comunicação \\
\hline
\end{tabular}




\begin{tabular}{|l|l|}
\hline $\begin{array}{l}\text { 11. Planejamento e } \\
\text { Solução }\end{array}$ & $\begin{array}{l}\text { Habilidades de resolução de problemas: Discutindo e descobrindo } \\
\text { maneiras de solucionar situações }\end{array}$ \\
\hline 12. Consolidação & Consolidação, feedback e entrega dos certificados de conclusão \\
\hline
\end{tabular}

A estrutura das sessões de desenvolvimento manteve-se constante ao longo do processo, marcada pelos seguintes passos:

1. Retomada da semana anterior e revisão do tema.

2. Introdução do assunto da semana, solicitando a participação dos usuários para trazerem exemplos práticos de sua vida, especificando quando, onde e como essa habilidade é usada ou requerida, quais as dificuldades e facilidades com relação à específica habilidade.

3. Ideias para superar essa dificuldade. Neste momento, pode-se optar por dividir o comportamento em componentes menores como forma de diminuir a ansiedade e auxiliar a desenvoltura dos usuários. Busca-se relação com aprendizados da semana anterior.

4. Role play (encenações de situações cotidianas em um ambiente controlado e assistido).

5. Debriefing (balanço da atividade, quando busca-se nomear e especificar observações do comportamento e sentimentos despertados, identificando aspectos produtivos e prejudiciais à comunicação, atentando para questões não verbais e para leitura de sinais ambientais que modulam e direcionam interações).

6. Consolidação do aprendizado.

Este trabalho é um relato de experiência, de forma que os resultados e discussão são baseados em registros e observações dos profissionais, fruto de atividades da assistência. Os princípios éticos de sigilo e confidencialidade foram respeitados, entretanto, não foi necessária tramitação da proposta em Comitê de Ética.

\section{Resultados}

No primeiro encontro, participaram 7 usuários. A atividade iniciou com a apresentação dos integrantes. Os coordenadores apresentaram a proposta do THS através da seleção de cartas do Baralho de Habilidades Sociais (RODRIGUES e FOLQUITTO, 2015) que demandam uma solução para situações sociais e permitem avaliar as habilidades sociais. Por exemplo, uma carta retratava uma consulta médica, em que familiar e médico discutiam sobre 
uma paciente sem incluí-la na discussão. Os usuários eram solicitados a dizer o que fariam nesta situação. A solução da usuária G foi: "Eu ficaria ansiosa, mas não falaria nada, isso já aconteceu comigo inclusive”. Nesse sentido, outra situação que demandava a resolução de um conflito entre irmãos, o usuário A sugere: "Para ele parar de brigar eu iria olhar feio para ele" apresentando uma solução bastante pueril.

Através da fala dos participantes, percebeu-se que compreenderam a proposta do grupo dentro de suas possibilidades: "Esse grupo é pra aprender a não ficar parecendo um cara esquisito" relatou B. Assim, identificaram-se as demandas do grupo e foi realizado um contrato com os usuários contemplando aspectos de sigilo e a importância de assiduidade. Os usuários no geral mostraram-se motivados para esta modalidade de trabalho verbalizando: "Fiquei feliz de ser selecionada acho que pode ser uma oportunidade para mim e também uma melhora para as pessoas com quem eu convivo", trouxe G.

No segundo encontro, havia seis participantes presentes. Os integrantes foram questionados a respeito de situações interpessoais nas quais tinham dificuldades. A situação mais recorrente foi a de resolução de conflitos familiares. Assim, foi possível formular os objetivos do grupo: melhorar habilidade de posicionar-se em relações familiares e buscar soluções mais assertivas na resolução de conflitos. Durante este levantamento, a angústia dos participantes relacionadas à comunicação ficou exemplificada no relato de C: "Eu nunca sei o que dizer, às vezes preferia ser mudo".

Diante do levantamento de aspectos introdutórios, as sessões foram mais direcionadas, com temáticas específicas. Na terceira sessão, trabalhou-se com comunicação inicial, contemplando a habilidade de fazer pedidos, aproximar-se dos outros e pedir informações na rua. Através de uma situação sugerida pelo usuário B, na qual perguntava a um estranho a localização de um cabeleireiro, foram trabalhados em grupo formas de tornar essa comunicação mais efetiva, delineando-se algumas estratégias como pensar bem na pergunta a fim de obter uma resposta com a informação completa e clara, observar a pessoa de quem desejam se aproximar a fim de avaliar se ela está disponível e apresentar-se de maneira educada e gentil.

Na quarta sessão, o objetivo foi trabalhar habilidades corporais enfatizando aspectos como habilidade de olhar-se e olhar os outros, postura, trejeitos e tiques. Para isso, o grupo utilizou a técnica de roleplay com a seguinte situação: um dos coordenadores e o participante A simularam uma entrevista de emprego para o cargo de repositor de estoque de supermercado. O coordenador foi o entrevistador e perguntou qual o objetivo do usuário com 
o emprego e a resposta de A foi: “Quero fazer amizade”. Após, o grupo que observou a cena apontou alguns aspectos que poderiam ser melhorados como a qualidade da resposta, entendendo que a resposta emitida não seria adequada para uma situação formal e para atingir o objetivo que era conseguir o emprego. Além disso, pontuou-se aspectos da postura do usuário que permaneceu a maior parte do tempo olhando para o chão.

Para qualificar a discussão, os coordenadores apresentaram a diferença de comunicação verbal e não verbal com exemplos de imagens em que, através da postura, era possível identificar situações de interesse ou tédio. O grupo aproveitou o espaço e apresentou algumas dúvidas como: "Se eu não souber o que responder e ficar nesse tempo pensando e olhando para o chão é ruim?" questionou E, e "Tem alguns tiques que não consigo controlar, o que eu poderia fazer?" indagou C. Trabalhou-se a diferença de tique e questões de sintomatologia associadas ao quadro psiquiátrico ou efeitos adversos da medicação e as possibilidades de intervenção nesse processo. Questões de ansiedade associadas a uma situação formal como entrevista de emprego também foram apontadas. Após a discussão, foi realizado o roleplay novamente e desta vez o usuário A buscou atentar para os aspectos que o grupo levantou e demonstrou postura mais adequada. Com relação ao objetivo do emprego, formulou a resposta de outra forma: "Preciso de dinheiro para ajudar minha família".

A quinta e a sexta sessões abordaram temáticas semelhantes: a habilidade de iniciar, continuar e finalizar conversas e leitura social. As duas propostas referem-se à capacidade de apreensão do ambiente e avaliação da disponibilidade do outro de iniciar uma conversação ou da intenção de interromper uma interação. Para isso, é importante que os participantes possuam capacidade de compreender sinais não verbais. Esta habilidade mostrou-se mais difícil de ser identificada considerando que muitas vezes estes sinais são apresentados de maneira sutil. O usuário A, por exemplo, por apresentar pensamento concreto, durante uma simulação, só compreendeu que a coordenadora não estava disponível para falar com ele, quando ela ficou olhando para os lados e para o relógio de maneira repetitiva. O grupo buscou nomear estratégias para identificar estes sinais e facilitar a abordagem assertiva dos usuários.

A sétima sessão utilizou como temática específica a habilidade de expressar sentimentos. Trabalhou-se reações e sentimentos esperados diante de determinadas situações, por exemplo, sentimentos de tristeza, angústia e/ou raiva podem emergir diante da morte de uma pessoa querida. O usuário A parte de uma premissa que todo sentimento que causa dor deve ser excluído/esquecido. Já o usuário E entende que "às vezes é normal ficar com raiva, eu fico com raiva do meu pai quando a gente briga”. Os coordenadores do grupo 
apresentaram algumas sugestões para lidar melhor com as emoções, como identificar e aceitar o que está sentindo, aprender a reconhecer como o corpo reage a determinados sentimentos e buscar formas alternativas de lidar com os sentimentos, como escrever sobre o que sente ou realizar exercícios físicos.

A oitava sessão trabalhou a habilidade fazer pedidos e expressar necessidades. O grupo trabalhou a partir de roleplay. No cenário, o usuário $\mathrm{D}$, que reside em uma pensão, vai até a proprietária do espaço e solicita: "Eu quero trocar de quarto, ir para o quarto maior". A proprietária responde que é possível, mas irá custar quinhentos reais a mais e o usuário concorda prontamente. Na discussão, o grupo sugere questionar por que esse quarto é tão mais caro, tentar diminuir o valor ou pedir um tempo para pensar sobre o assunto e discutir com outras pessoas. Entretanto, no segundo momento da atuação, não houve diferença significativa na resposta de D. O usuário se dá conta de sua dificuldade em flexibilizar e mudar padrões de resposta, o que pode associar-se a limitação cognitiva e/ou a questões de ansiedade.

A nona sessão abordou habilidades necessárias para fazer e ouvir críticas. Foi enfatizada a diferença entre crítica construtiva e destrutiva. No roleplay, a usuária G precisava dizer a um amigo que parasse de fumar e constrói o seguinte relato: "você poderia utilizar um adesivo ou chiclete, existem grupos também”. O grupo entendeu que ela foi efetiva na orientação e apresentou uma crítica construtiva. Já os usuários A e D apresentaram dificuldades em apresentar argumentos para fazer as críticas de forma adequada. Em ambos os casos, associou-se a dificuldade cognitiva para compreensão de questões mais elaboradas.

A décima sessão, ao abordar a limpeza da comunicação, retomou conceitos gerais dos encontros anteriores. Realizou-se simulação realística no CAPS: a partir da necessidade real de um usuário, expressada através de pergunta ou pedido, ele era estimulado a fazer a questão para um membro da equipe assistencial. O processo foi observado por um dos coordenadores e, após, realizou-se em grupo uma discussão sobre a assertividade do usuário. Antes de iniciar, o grupo buscou relembrar aspectos importantes a serem observados: postura, tom de voz, leitura social, como iniciar e terminar uma conversa.

A décima primeira sessão abordou resolução de problemas. Nesta sessão, foram utilizadas novamente as cartas com situações-problemas do Baralho de Habilidades Sociais (RODRIGUES e FOLQUITTO, 2015) para, de alguma forma, tentar avaliar mudanças nos repertórios relacionados às habilidades sociais dos participantes. A usuária G retirou a mesma 
carta da primeira sessão e trouxe uma solução mais assertiva: "Eu perguntaria o que significa isso que ele está dizendo, ele tem que me explicar né”.

A última sessão buscou retomar aspectos discutidos ao longo do treinamento. Os participantes verbalizaram que o grupo foi muito importante e que agora possuem maior repertório de estratégias para lidar com situações de interação social. Posteriormente, foi entregue um certificado de conclusão para cada usuário e os coordenadores enfatizaram pontos positivos da evolução de cada um.

\section{Discussão}

De maneira geral, observou-se crescente motivação dos usuários, possivelmente relacionada a elementos como coesão e desenvolvimento de identidade grupal. Entretanto, percebeu-se oscilação quanto a frequência dos usuários ao longo dos encontros, com média de 4 a 5 participantes por sessão. Somado a isso, ressalta-se que dois usuários, C e E, apresentavam atrasos frequentes. Identificou-se que as diferenças na capacidade cognição impactaram o andamento do grupo.

Inicialmente, a motivação para participação nas atividades partia predominantemente dos coordenadores, os quais precisavam ser criativos, ativos e dinâmicos. Os usuários mantinham postura passiva e retraída, o que pode ser reflexo de anos em tratamentos assistenciais em modelos ultrapassados. Até meados da década de 70, o modelo assistencial ao doente mental no Brasil era asilar e hospitalar, com destituição da capacidade decisória, cidadã e ativa dos pacientes (JORGE et al., 2011). Esta realidade, entretanto, vem mudando desde a Reforma Psiquiátrica e a promulgação da Lei 10.216 (BRASIL, 2001), a qual busca o respeito e a garantia de direitos para indivíduos com transtorno mental.

Entende-se que houve um aumento da motivação por parte dos usuários diante da vinculação com o grupo e por sentirem-se acolhidos neste espaço. Dispositivos relacionais, como vínculo, acolhimento e co-responsabilização, convocam os usuários a posicionarem-se de maneira ativa na construção do processo de adoecimento/tratamento/cura. O certificado de conclusão, ao final do processo, representa, de maneira concreta, uma conquista simbólica de autonomia, responsabilidade e comprometimento. O fato de ser um grupo fechado cria uma identidade de grupo e favorece o vínculo entre os integrantes, possibilitando a expressão de dúvidas, angústias e sentimentos, o que é fundamental quando se pensa em treinamento de habilidades sociais. 
No grupo de THS realizado, foi observado impacto da expressão da sintomatologia dos participantes na adesão ao grupo. Como exemplo, sintomas de Transtorno Obsessivo Compulsivo (TOC) apareceram nos atrasos e faltas. Essa realidade foi acolhida e trabalhada pelo grupo ao longo do processo, pois foi compreendido como sintoma e não como resistência dos participantes. De acordo com MITSI (2004), os comportamentos obsessivo-compulsivos podem perturbar gravemente o desempenho diário dos indivíduos com TOC, gerando um repertório social bastante limitado. O prejuízo nos relacionamentos se dá pela percepção do contato social como ameaçador e pelos longos períodos dedicados à execução de rituais e planejamento de meios para esquiva de estímulos eliciadores de ansiedade. No caso dos participantes do grupo, a possibilidade de identificar e diferenciar aspectos específicos da sintomalogia do transtorno psiquiátrico de dificuldades individuais associadas a habilidades sociais pode delinear objetivos mais concretos para esses usuários.

A coesão e homogeneidade do grupo favoreceram o funcionamento grupal, tendo uma relação direta com as metas e finalidades a serem alcançadas (SANTOS, 2010). No THS, as dificuldades e situações problemas devem emergir da realidade dos usuários para que o treino possa ser mais facilmente aplicado no cotidiano (BELLAK et al., 2004; CABALLO, 2007). Indivíduos da mesma faixa etária tendem a partilhar os mesmos interesses ou preocupações. Isto ficou aparente no grupo através da discrepância entre interesses e vivências da usuária $\mathrm{F}$, única integrante com 57 anos em grupo de jovens adultos e que, por vezes, apresentava dificuldade em envolver-se e relacionar suas experiências e conflitos com as dos outros usuários. Sabe-se que, quanto mais próximo o roleplay for da realidade do indivíduo, mais provável que este consiga colocar o aprendizado em prática no seu cotidiano (KOPELOWICZ, LIBERMAN e ZARATE, 2006).

A discrepância na capacidade cognitiva de alguns usuários também afetou a coesão e funcionamento grupal. Apesar de os usuários A e D terem tido benefício na participação do grupo, avalia-se que, em alguns momentos, suas dificuldades cognitivas prejudicaram o andamento do THS. Apesar de terem indicação para este tipo de treinamento, o método utilizado exigia uma compreensão além de suas capacidades. Apesar dos entraves, o grupo demonstrou capacidade de acolhimento e adaptação, permitindo repetições e retomadas das habilidades aprendidas, as quais foram trabalhadas lentamente.

As mudanças de atitudes foram percebidas através de relatos dos próprios usuários e familiares, observações da equipe em diferentes atividades do CAPS e dos exercícios realizados no THS durante o grupo. Como exemplo, conforme apresentado nos resultados, a 
usuária $\mathrm{G}$ obteve mudança significativa de atitude diante da mesma situação-problema. No início, apresentou atitude caracterizada por passividade e retraimento; já ao fim do THS, frente à mesma situação, a usuária demonstrou postura proativa e assertiva. Essa mudança é um dos principais objetivos desta intervenção: melhorar a assertividade, buscando a expressão de dúvidas ou pensamentos de forma clara e objetiva e, ao mesmo tempo, considerando o contexto e receptor (DEL PRETTE \& DEL PRETTE, 2011).

A equipe assistencial, que acompanha os usuários diariamente, percebeu mudanças nas atitudes dos participantes, possivelmente associada ao desenvolvimento da habilidade de leitura social. Como exemplo, usuários que costumavam interromper consultas de outros ou discussões de equipe, conseguiram, ao final do THS, sinalizar a necessidade de falar e aguardar o momento adequado. A generalização do aprendizado é uma das principais preocupações do THS. Usuários devem ser capazes de, gradativamente, aplicar as habilidades desenvolvidas em suas realidades (KOPELOWICZ, LIBERMAN e ZARATE, 2006; CABALLO, 2007). Grande parte do cotidiano e das relações sociais dos usuários do CAPS ocorrem nesta instituição. Dessa forma, é relevante que os profissionais puderam perceber diferenças na conduta e atitude dos usuários, possivelmente associadas às habilidades desenvolvidas através do THS.

\section{Considerações finais}

A realização da proposta de THS com usuários com transtorno mental grave em um CAPS mostrou-se efetiva, atingindo os objetivos propostos. O modelo de grupo, fechado, com perfil para ingresso e com número determinado de sessões, contribuiu para coesão e identidade grupal, assim como para a criação de vínculo entre os integrantes, aspectos essenciais para o sucesso da intervenção. A proatividade dos coordenadores, associada à convocação da participação ativa dos usuários através de exemplos e vivências de sua realidade, permitiu que a atividade fosse desenvolvida com êxito.

Entretanto, algumas limitações influenciaram no desenvolvimento do grupo. Entre elas, destaca-se a diferença na idade e na capacidade cognitiva dos participantes. Sugere-se a oferta de diferentes modalidades de THS, adaptadas para o público específico a fim de que o benefício seja o maior possível.

O treinamento de habilidades sociais é relevante para a prática clínica no contexto da saúde mental coletiva. Uma das principais funções do CAPS é facilitar e estimular a autonomia, cidadania e reinserção social dos indivíduos. Práticas inovadoras são necessárias a 
fim de que os usuários permaneçam motivados a seguir o tratamento e que se sintam vistos e valorizados em um contexto de vida que, na maior parte dos casos, têm a exclusão social e a discriminação como características intrínsecas à sua condição.

\title{
GRUPO DE ENTRENAMIENTO EN HABILIDADES SOCIALES EN UN CENTRO DE ATENCIÓN PSICOSOCIAL
}

\section{Resumen}

La asistencia al usuario con trastorno mental en Brasil está pasando por diversas reformulaciones. Actualmente, ese público es atendido en un Centro de Atención Psicosocial (CAPS) por un equipo multiprofesional que realiza el cuidado a través de diferentes propuestas terapéuticas. Este trabajo busca presentar una experiencia de un grupo deentrenamiento en habilidades sociales (EHS) en un CAPS. El EHS ha sido desarrollado con 7 usuarios con trastorno mental grave, ha tenido uma duración de 12 sesiones basadas en un cronograma de temáticas específicas y ha sido coordinado por dos profesionales de enfermería y psicología. Este estudio es un relato de experiencia basado en los registros y observaciones de los profesionales. La participación de los usuarios en el grupo ha permitido el desarrollo de habilidades como autonomía, responsabilidad y asertividad frente a situaciones sociales. Las características del grupo cerrado y perfil semejante han permitido una vinculación buena de los participantes y la formación de una identidad grupal, características fundamentales para la propuesta EHS. Como factores limitadores se ha percibido la diferencia en la capacidad cognitiva de los participantes que exigieron una flexibilización de algunas propuestas. La realización de la propuesta EHS, con usuarios con trastorno mental grave, en un CAPS, se ha mostrado efectiva, alcanzando los objetivos propuestos y configurándose como uma práctica clínica innovadora en el contexto de salud mental colectiva.

Palabras-Clave: Habilidades Sociales; Salud Mental; Servicios de Salud Mental;

\section{SOCIAL SKILLS TRAINING AT A REHABILITATION FACILITY FOR INDIVIDUALS WITH SEVERE MENTAL ILLNESS}

\begin{abstract}
The treatment for people with psychiatric disabilities has been changing in Brazil. Currently, the care is provided by a multidisciplinary team at rehabilitation facilities called Centro de Atenção Psicossocial (CAPS). This article intends to describe the experience of a Social Skills Training (SST) group conducted at a CAPS. Seven individuals with severe mental illness participated in the SST, which was organized in 12 planned and structured sessions and coordinated by a Psychologist and a Nurse. This is an experience report based on the notes and observation of these professionals. Participants developed abilities related to autonomy, responsibility and assertiveness. Being a closed and homogenous group facilitated the bond among participants and the consolidation of a group identity. Differences in cognitive ability hindered the process and demanded more flexibility and time in some tasks and activities. The SST is an effective psychosocial treatment for individuals with severe mental illness. This intervention is considered an innovative clinical practice in the mental health field in Brazil.

Key-Words: Social Skills, Mental Health; Mental Health Services;
\end{abstract}




\section{REFERÊNCIAS}

AMARANTE, Paulo. Saúde mental e atenção psicossocial. Rio de Janeiro: Fiocruz, 2007.

BELLACK, A. S. et al. Social skills training for schizophrenia: a step-by-step guide ( $2^{\mathrm{a}}$ ed.). New York: Guilford Press; 2004.

BRASIL. Lei 10.216 de 6 de abril de 2001. Dispõe sobre a proteção e os direitos das pessoas portadoras de transtornos mentais e redireciona o modelo assistencial em saúde mental. Diário Oficial da União. Disponível em:

http://www.planalto.gov.br/ccivil_03/leis/LEIS_2001/L10216.htm Acesso em: 01 de junho de 2020.

BRASIL. Ministério da Saúde. Secretaria de Atenção à Saúde. Departamento de Ações Programáticas Estratégicas. Saúde mental no SUS: os centros de atenção psicossocial. Brasília: Ministério da Saúde. Disponível em: http://www.ccs.saude.gov.br/saude_mental/pdf/sm_sus.pdf Acesso em: 01 de junho de 2020.

CABALLO, E. Vicente. Manual para o tratamento cognitivo-comportamental dos transtornos psicológicos da atualidade. São Paulo: Editora Santos, 2007.

DEL PRETTE, A. e DEL PRETTE, Z. A. P. Habilidades sociais: Intervenções efetivas em grupo. São Paulo: Casa do Psicólogo, 2011.

JORGE, M. S. B. et al. Promoção da Saúde Mental-Tecnologias do Cuidado: vínculo, acolhimento, co-responsabilização e autonomia. Ciência \& Saúde Coletiva, v.16, n.7, p. 30513060, jan-jul 2011. Disponível em:

http://www.scielo.br/scielo.php?script=sci_arttext\&pid=S1413-81232011000800005 Acesso em: 01 de junho de 2020.

KOPELOWICZ, A., LIBERMAN, R. P. e ZARATE, R. Recent advances in social skills training for schizophrenia. Schizophrenia bulletin, v.32, n. 1, p. 12-23, 2006. Disponível em: https://www.ncbi.nlm.nih.gov/pmc/articles/PMC2632540/ Acesso em: 01 de junho de 2020.

MITSI, C. A., SILVEIRA, J. M. e COSTA, C. E. (2004). Treinamento de habilidades sociais no tratamento do transtorno obsessivo-compulsivo: um levantamento bibliográfico. Revista Brasileira de Terapia Comportamental e Cognitiva. São Paulo, v. 6, n. 1, p. 49-59, junho 2004 . Disponível em: http://pepsic.bvsalud.org/scielo.php?script=sci_arttext\&pid=S151755452004000100006\&lng=pt\&nrm=iso. Acesso em: 01 de junho de 2020.

RODRIGUES, L. C. e FOLQUITTO, T. C. Baralho de Habilidades Sociais. Novo Hamburgo, RS: Ed. Synopsis, 2015.

SANTOS, E. G. O grupo como estratégia terapêutica nos centros de Atenção Psicossocial Álcool e Drogas do Espírito Santo. 2010. 123f. Dissertação de mestrado, Universidade Federal do Espírito Santo, Espírito Santo, Brasil, 2010.

SADOCK, B. J., SADOCK, V. A. e RUIZ, P. Compêndio de Psiquiatria: Ciência do Comportamento e Psiquiatria Clínica. Porto Alegre: Artmed, 2010. 
Data de recebimento: 20/06/2018

Data de aceite: 04/06/2020

\section{Sobre as autoras:}

Nilve Junges é Psicóloga, Especialista em Saúde Mental pelo Hospital de Clínicas de Porto Alegre e Especialista em Psicologia da Saúde pelo Conselho Federal de Psicologia (CFP).

Flavia Pimentel Pereira é Enfermeira do Serviço de Enfermagem Psiquiátrica do Hospital de Clínicas de Porto Alegre (HCPA), Chefe da Unidade Centro de Atenção Psicossocial do HCPA, Mestre em Saúde Mental pela Universidade Federal do Rio Grande do Sul (UFRGS) e Especialista em Saúde Mental pela UFRGS.

Juliana Unis Castan é Psicóloga do Hospital de Clínicas de Porto Alegre (HCPA), Mestre em Counseling and Personnel Services (University of Maryland, College Park, EUA) e Especialista em Psicologia Hospitalar pelo Conselho Federal de Psicologia (CFP). 